\title{
Allowable deviation of LC layer thickness in cholesteric LCDs
}

\author{
A. Rybalochka ${ }^{1)}$, V. Sorokin ${ }^{2}$, A. Sorokin ${ }^{3)}$ \\ Institute of Semiconductor Physics, NAS of Ukraine, 45 pospect Nauky, 03028 Kyiv, Ukraine \\ Phone: +380 (44) 265 9786; fax: +380 (44) 265 5785: \\ ${ }^{1)}$ e-mail: a_ryb@isp.kiev.ua \\ 2)e-mail:vsorokin@isp.kiev.ua \\ ${ }^{3)}$ e-mail: asorokin@isp.kiev.ua
}

\begin{abstract}
The deviation of liquid crystal (LC) layer thickness in a display cell has considerable influence on the image quality at the addressing of cholesteric liquid crystal displays (ChLCD) by different kind of dynamic drive schemes. The driving principles that are realized in dynamic drive schemes by sequences of voltage pulses apply certain limitations on the value of this deviation. In this paper, we have compared allowable relative deviation of the LC layer thickness for two simple two-level dynamic drive schemes in ChLCD by the "dynamic hysteresis" method.
\end{abstract}

Keywords: cholesteric liquid crystal, cholesteric display, drive scheme.

Paper received 24.01.02; revised manuscript received 05.02.02; accepted for publication 12.02.02.

\section{Introduction}

The researcher's interest to ChLCD had been attracted due to its unique properties such as bistability, low power consumption, wide viewing angles, polarizers free, high contrast ratio, technological simplicity and possibility of information representation with high information content. Effect of bistability in cholesteric liquid crystals (CLC) lies in the presence of two stable and optically contrasting states at zero field: the planar state and the focal conic state [1]. In the planar state the helical axis of ChLC are more or less perpendicular to the surface cell, and the liquid crystal Bragg reflects light peaked at the wavelength given by $l_{0}=n P_{0}$, where $P_{0}$ is the pitch of the liquid crystal helix and $n$ is the average reflection index. In the focal conic state, the helical axes are more or less parallel to the cell surface. Incident light is weakly scattered and absorbed by black background of display. Thereby pixels with CLC in focal conic state look black.

The way of the transition between these two stable states of the ChLC is well known [2]. The ChLC is transformed from the planar state to the focal conic state by low-voltage pulses and if stabilized, it remains in the stable focal conic state for a long time. For the transition of the ChLC from the focal conic state to the planar state, at first, the cholesteric helix must be unwound applying the high-voltage pulses. This state of the ChLC is called the homeotropic state $(\mathrm{H})$, and the transition of the $\mathrm{ChLC}$ to this state is called as the cholesteric-nematic transition. The cholesteric-nematic transition has the hysteresis character. From the homeotropic state the ChLC can be addressed to either planar or focal conic stable states. If the high voltage is switched to a low voltage level, the ChLC transforms to the planar state. If the high voltage is switched to a middle voltage level, the ChLC transforms to the focal conic state. It was studied that the transition of the ChLC from the homeotropic state to the planar state occurs through the transient planar state with the pitch of the ChLC $P^{*} \approx 2 P_{0}$ [3]. This state is unstable. Also there is the range of voltage levels where the field induced homeotropic state is hold, and the transient planar state is transformed to the focal conic state by the same voltage level. These specific properties of the ChLC are used in the number of the dynamic drive schemes (DDS) for ChLCDs [4-9] 


\section{A. Rybalochka et al.:Permissible deviation of LC layer thickness in Cholesteric LCDs}

The simplest voltage waveforms at the addressing of the ChLCD with high multiplexing level are realized in the DDS “ $U / \sqrt{2}$ " [7] and the DDS “ $U / \sqrt{3 / 2}$ "[9]. Sequences of driving voltage pulses for these DDSs consist of pulses of two voltage levels only: $U$ and zero. Such driving sequences give possibility for using of simple and cheap drivers.

In cholesteric displays the LC layer thickness is about several micrometers. Therefore display cells are manufactured with high precision of the distance between glass plates. The higher requirements to the deviation of the display cell thickness the more cost and complexity of technology of display cell manufacturing. Consequently, using drive scheme that allow address displays with high allowable deviation of LC layer thickness can significantly decrease the cost of ChLCD.

In this work, an allowable relative deviation of LC layer thickness for the DDS " $U / \sqrt{2}$ " and the DDS " $U / \sqrt{3 / 2}$ " was experimentally determined and compared by the "dynamic hysteresis" method [10]. We performed our measurement on the display cell with thickness $\mathrm{d}=4.4 \mu \mathrm{m}$ at multiplexing level $N=240$ (QVGA LCD) with mixture of nematic liquid crystal MLC4900-100 $(84 \%)$ and $l$-menthol derivatives $(16 \%)$ that posses high twisting ability.

\section{Simple Two-Level DDSs}

For the DDSs " $U / \sqrt{2}$ " and " $U / \sqrt{3 / 2}$ " a schematic diagram of the effective voltage levels on the pixels from $m$-th current row for a display with $\mathrm{N}$ rows is presented in Fig.1.

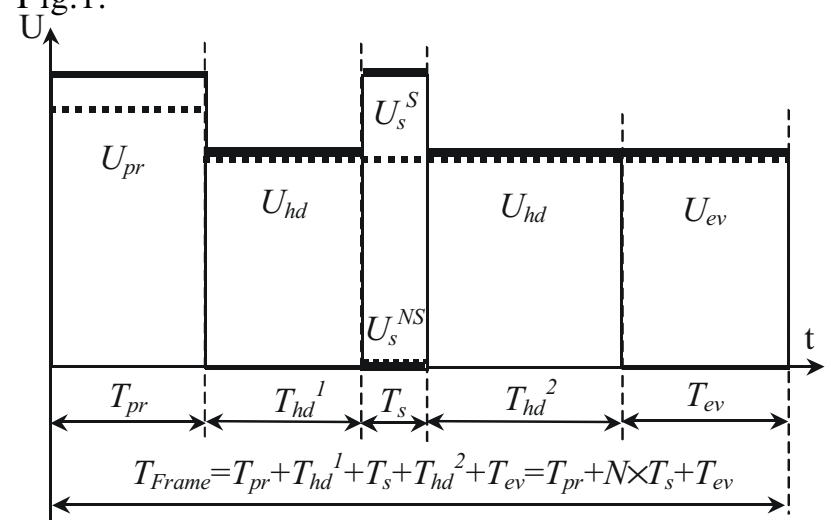

Fig. 1. The schematic diagram of the effective voltage levels on pixels for the DDS “ $U / \sqrt{2}$ " (solid line) and for the DDS " $U / \sqrt{3 / 2} "$ (dot line).

Effective voltage levels on the pixel are realized by applying to the row and column of LCD of two level pulses voltage with different form. This diagram shows that the drive voltage for both DDS can be subdivided into four stages: preparation, selection, holding and evolution. These DDSs are differed in the value of the effective voltage levels during different stages of the addressing. However driving ideology is the same for both DDSs. At the beginning of the addressing during the preparation stage $\left(T_{p r}\right)$, the preparation voltage $U_{p r}$ is applied to all dis- play pixels. The value of $U_{p r}$ must be enough to transform the ChLC into the homeotropic state, independent of its initial state. The first part of the holding stage follows the preparation stage and is intended to keep the ChLC in the homeotropic state by the holding voltage $U_{h d}$ during the time $T_{h d}{ }^{l}=(m-1) \times T_{s}$, where $T_{s}$ is a duration of the selection stage. During the selection stage the final state of the ChLC is determined. The voltage level $U_{S}^{S}$ keeps the ChLC in the homeotropic state in selected pixels. The voltage level $U_{s}{ }^{N S}$ lets the ChLC relaxation to the transient planar state in non-selected pixels. During the second part of the holding stage $\left(T_{h d^{2}}=(N-m) \times T_{s}\right)$ and the evolution stage $\left(T_{e v}, U_{e v}=U_{h d}\right)$, the ChLC remains in the homeotropic state, if it was kept in this state during the selection stage. In the case when the ChLC is relaxed to the transient planar state during the selection stage, it evolves to the focal conic state. After removal of voltage, the $\mathrm{ChLC}$ in selected pixels relaxes from the homeotropic state through the transient planar state into the stable planar state. In non-selected pixels the ChLC stays in the stable focal conic state. For these both DDS the full frame time of the addressing for a display with multiplexing level $N$ is $T_{\text {Frame }}=T_{p r}+T_{h d}+T_{s}+T_{e v}=T_{p r}+(N-1) T_{s}+T_{s}+T_{e v}=$ $=T_{p r}+N \times T_{s}+T_{e v}$.

For both DDSs real sequences of driving voltage pulses consist of pulses of two voltage levels: $U$ and zero. Proportions between effective voltage levels in different stages of the addressing are: for the DDS " $U / \sqrt{2} "-U_{p r}=U$, $U_{h d}=U_{e v}=U / \sqrt{2}, U_{s}{ }^{S}=U$ and $U_{s}{ }^{N S}=0$ (solid thick lines on Fig. 1); for the DDS " $U / \sqrt{3 / 2} "-U_{p r}=U, U_{h d}=U_{e v}=U /$ $\sqrt{3 / 2}, U_{s}^{S}=U / \sqrt{3 / 2} /$ and $U_{s}^{N} S_{=0}$ (dot thick lines on Fig.1). For the DDS " $U / \sqrt{3 / 2}$ " the maximal pulse voltage level $(U)$ in the drive sequences is less than for DDS " $U / \sqrt{2}$ " on 13 percents.

\section{Results and discussion}

For the determination of the maximal allowable relative deviation of LC layer thickness $\left(D_{p}\right)$ in the display cell, we used the "dynamic hysteresis" method [10]. This method consists in the following. At the addressing of the passive matrix ChLCD by the DDS " $U / \sqrt{2}$ " and the DDS " $U / \sqrt{3 / 2}$ ", sequences of driving voltage pulses for selected and non-selected pixels from any row of the display differ only in voltage level during selection stage and its arrangement concerning other stages. Realized during addressing transitions of ChLC between various states in all display pixels allow to note the following fact. If parameters of a drive scheme, such as the duration of preparation, selection and evolution stages, multiplexing level $N$ and voltage level $U$, provide addressing of the information in pixels from the first and the last rows of the display, these parameters will ensure also addressing of all others pixels in the display. Therefore, in the "dynamic hysteresis" method, only behavior of pixels from the first and the last row of the display is analyzed. In Fig. 2, the dependence of contrast ratio versus voltage level $(U)$ for pixels from the first $(m=1)$ and 


\section{A. Rybalochka et al.:Permissible deviation of LC layer thickness in Cholesteric LCDs}

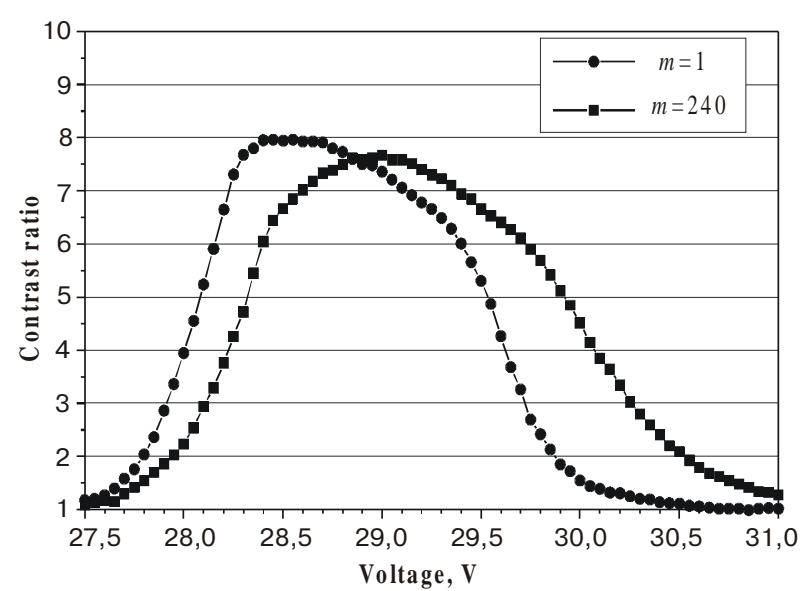

Fig. 2. The dependence of the contrast ratio versus the voltage level $(U)$ for the first $(m=1)$ and the last $(m=240)$ row of the display for the DDS “ $U / \sqrt{3 / 2}$ “.

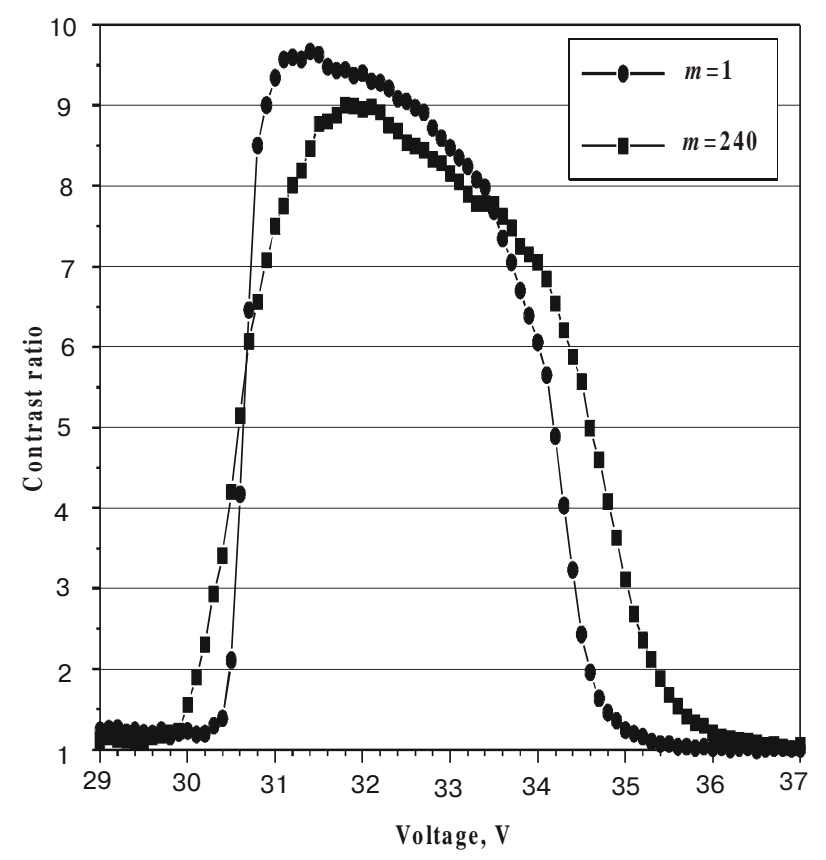

Fig. 3. The dependence of the contrast ratio versus the voltage level $(U)$ for the first $(m=1)$ and the last $(m=240)$ row of the display for the DDS “ $U / \sqrt{2}$ “.

the last $(m=240)$ rows of the display for the DDS " $U / \sqrt{3 / 26}$ is shown. Analogous dependence for the DDS " $U / \sqrt{2}$ " is shown in Fig. 3. Duration of the preparation, the evolution and the selection stages were selected identical for both DDS: $T_{p r}=T_{e v}=100 \mathrm{~ms}, T_{s}=3 \mathrm{~ms}$. There is a range of voltage for any contrast ratio, at which display pixels are addressed with contrast ratio not less than chosen level. For example for contrast ratio $\mathrm{C}=7$ for the $\mathrm{DDS}$ " $U / \sqrt{2}$ ", the range is determined by voltage levels $U_{\min }=$ $30.9 \mathrm{~V}$ and $U_{\max }=33.7 \mathrm{~V}$, and for the DDS " $U / \sqrt{3 / 2}$ " this range is determined by voltage levels $U_{\text {min }}=28.6 \mathrm{~V}$ and $U_{\max }=29.1 \mathrm{~V}$. These voltage levels allow to calculate maximal relative deviation of LC layer thickness,

SQO, 5(1), 2002 at which the given display will be addressed with contrast ratio not less than $\mathrm{C}=7$, using the following expression [10]:

$$
D p= \pm \frac{U \max -U \min }{U \max +U \min } \times 100 \%
$$

Thus, we have obtained the following result: the maximal relative deviation of LC layer thickness, at which information on the display will be addressed with contrast ratio not less than $\mathrm{C}=7$ for the DDS " $U / \sqrt{2}$ " $D_{p}= \pm 4.3 \%$, and for the DDS “ $U / \sqrt{3 / 2}$ " $-D_{p}= \pm 0.9 \%$. The voltage levels $U_{\min }$ and $U_{\max }$ for several values of contrast ratio for the DDS " $U / \sqrt{2}$ " and the DDS " $U /$ $\sqrt{3 / 2}$ " are given in Table 1 . Dependences of the parameter $D_{p}$ versus contrast ratio C for both DDS are shown in Fig. 4. From presented data in Table 1 and Fig. 4, we come to following conclusions. For all contrast levels a value of the parameter $D_{p}$ for the DDS " $U / \sqrt{2}$ " always exceeds the value of the parameter $D_{p}$ for the DDS " $U /$ $\sqrt{3 / 2}$ " more than by 2.5 times. Besides, maximal contrast ratio for the DDS " $U / \sqrt{2}$ " and DDS "U/ $\sqrt{3 / 2}$ " respectively.

Table 1. The voltage levels $U_{\min }$ and $U_{\max }$ for several values of the contrast ratio for the DDS " $U / \sqrt{2}$ " and the DDS " $U / \sqrt{3 / 2}$ ".

\begin{tabular}{c|c|ccccc}
\hline \hline \multirow{2}{*}{ D D } & \multirow{2}{*}{ Voltage } & \multicolumn{5}{|c}{ Contrast ratio } \\
\cline { 3 - 7 } & & 8 & 7 & 6 & 5 & 4 \\
\hline \multirow{2}{*}{$" U / \sqrt{2} "$} & $U_{\min }, \mathrm{V}$ & 31.2 & 30.9 & 30.7 & 30.7 & 30.6 \\
\cline { 2 - 7 } & $U_{\max }, \mathrm{V}$ & 33.1 & 33.7 & 34.0 & 34.1 & 34.3 \\
\hline \multirow{2}{*}{$" U / \sqrt{3 / 2} "$} & $U_{\min }, \mathrm{V}$ & - & 28.6 & 28.4 & 28.4 & 28.3 \\
\cline { 2 - 7 } & $U_{\max }, \mathrm{V}$ & - & 29.1 & 29.4 & 29.5 & 29.6 \\
\hline \hline
\end{tabular}

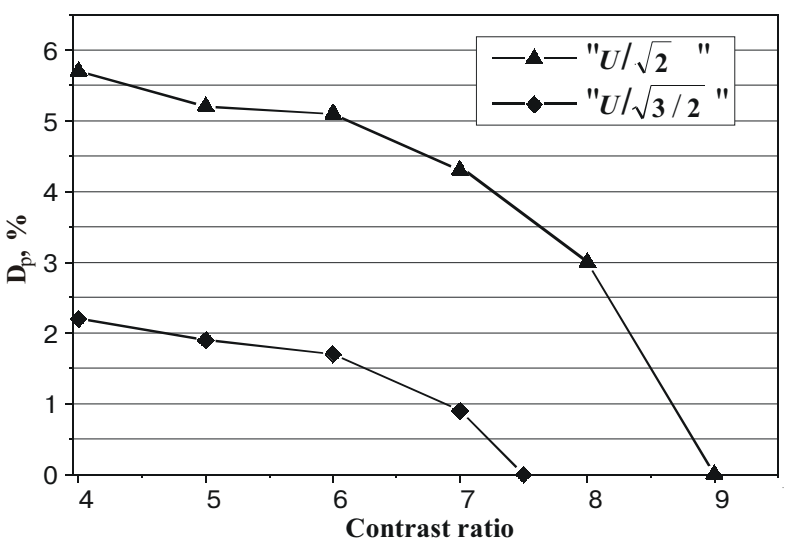

Fig. 4. Dependences of the permissible relative deviation of LC layer thickness $\left(D_{p}\right)$ versus the contrast ratio for the DDS " $U / \sqrt{2}$ " and the DDS " $U / \sqrt{3 / 2}$ ".

\section{Conclusion}

In this paper, using the example of the cholesteric display for two driving methods (DDS " $U / \sqrt{2}$ " and " $U / \sqrt{3 / 2}$ "), we determined dependences of maximal permissible deviation in LC layer thickness in ChLCD versus contrast ratio. Duration of preparation, evolution and selection stages and multiplexing level were selected 


\section{A. Rybalochka et al.:Permissible deviation of LC layer thickness in Cholesteric LCDs}

identical for both DDS: $T_{p r}=T_{e v}=100 \mathrm{~ms}, T_{s}=3 \mathrm{~ms}$ and $N=240$. The values of voltage levels that define parameter $D_{p}$ exceed analogues voltage levels for the DDS " $U / \sqrt{3 / 2}$ " by $2-5 \mathrm{~V}$. However, values of the parameter $D_{p}$ for the DDS " $U / \sqrt{2}$ " exceed similar values of this parameter for the DDS “ $U / \sqrt{3 / 2}$ "by more than 2.5 times. Besides the maximal value of contrast ratio obtained in ChLCD for the DDS " $U / \sqrt{2}$ " is $\mathrm{C}=9$, and for the DDS " $U / \sqrt{3 / 2}$ " is $\mathrm{C}=7.5$. The obtained results show that from the viewpoint of requirements to the value of voltage level $U$ the DDS " $U / \sqrt{3 / 2}$ " is better. However, to reach higher levels of an image contrast and more considerable maximal deviations in LC layer thickness at high multiplexing levels, the DDS “ $U / \sqrt{2}$ " is better. Thus, the presence of two simple two-level dynamic drive schemes for bistable ChLCD, which allow to vary both parameter $D_{p}$ and contrast of the image only by changing of a voltage level $U$, can appear useful for application in passive matrix addressing of ChLCD.

\section{Acknowledgements}

The authors would like to gratitude Mr P. Titarenko and $\mathrm{Mr} \mathrm{Yu}$. Kolomzarov for their help in the preparation of liquid crystal display cells and $\mathrm{Mr} \mathrm{V}$. Nazarenko for his assistance in the preparation of LC materials.

\section{References}

1. D. K. Yang, J. L. West, L.-C. Chien and J. W. Doane, Control of Reflectivity and Bistability in Displays Using Cholesteric Liquid Crystals // J. Appl. Phys. 76 (2), pp. 1331-1333 (1994).

2. X. Y. Huang, J. W. Doane, D. K. Yang, The Transient Response and Dynamic Drive of Cholesteric Liquid Crystal Displays // Journal of the SID 5/3, pp. 179-187 (1997).

3. D. K. Yang, Z. J. Lu, Switching Mechanism of Bistable Reflective Cholesteric Displays // SID 95 Digest, pp.351-354 (1995).

4. X. Y. Huang, D. K. Yang, P. Bos and J. W. Doane, Dynamic Drive for Bistable Reflective Cholesteric Displays: A Rapid Addressing Scheme // SID 95 Digest, pp.347-350 (1995).

5. X. Y. Huang, M. Stefanov, High-performance Dynamic Drive Scheme for Bistable Reflective Cholesteric Displays // SID 96 Digest, pp.359-362 (1996).

6. M. Zhu, D. K. Yang, High-Speed Dynamic Drive Scheme for Bistable Reflective Cholesteric Displays // SID 97 Digest, pp.97-100 (1997).

7. V. Sorokin, Simple Driving Methods for Cholesteric Reflective LCDs // Asia Display'98, pp. 749-752 (1998).

8. A. Rybalochka, V. Sorokin, S. Valyukh, A. Sorokin, V.Nazarenko, Dynamic Drive Scheme for Fast Addressing of Cholesteric Displays // SID 2000 Digest, pp. 818-821 (2000).

9. A. Rybalochka, V. Sorokin, S. Valyukh, A. Sorokin, Simple Drive Scheme for Bistable Cholesteric LCDs // SID 2001 Digest, pp. 882-885 (2001).

10. A. Rybalochka, V.Sorokin, S. Valyukh, Determination of maximum permissible relative deviation of LC layer thickness in dynamically addressed ChLCD with high information content // Proceeding of VII-th International Symposium “Advanced Display Technologies”, pp.125-128 (1998). 